\title{
Percolation parameter and percolation-threshold estimates for 3D random ellipses with widely-scattered distributions of eccentricity and size
}

\author{
J.-R. de Dreuzy, P. Davy and O. Bour \\ Géosciences Rennes (UPR 4661) Campus de Beaulieu, 35042 Rennes Cédex, France \\ Corresponding author: O. Bour. E-mail: bour@univ-rennes1.fr
}

\begin{abstract}
In fractured materials of very low matrix permeability, fracture connectivity is the firstorder determinant of the occurrence of flow. For systems having a narrow distribution of object sizes (short-range percolation), a first-order percolation criterion is given by the total excluded volume that is almost constant at threshold. In the case of fractured media, recent observations have demonstrated that the fracture-length distribution is extremely large. Because of this widely-scattered fracture-length distribution, the classical expression of the total excluded volume is no longer scale invariant at the percolation threshold and has no finite limit for infinitely large systems. Thus, the classical estimation method of the percolation threshold established in short-range percolation becomes useless for the connectivity determination of fractured media. In this study, we derive a new expression of the total excluded volume that remains scale invariant at the percolation threshold and that can thus be used as the proper control parameter, called parameter of percolation in percolation theory. We show that the scale-invariant expression of the total excluded volume is the geometrical union normalized by the system volume rather than the summation of the mutual excluded volumes normalized by the system volume. The summation of the mutual excluded volume (classical expression) remains linked to the number of intersections between fractures, whereas the normalized geometrical union of the mutual excluded volume (new expression) can be basically identified to the percolation parameter. Moreover, fluctuations of this percolation parameter at threshold with length and eccentricity distributions remain limited within a range of less than one order magnitude, giving in turn a first rough percolation criterion. We finally show that the scale-dependence of the percolation parameter causes the connectivity of fractured media to increase with scale, meaning especially that the hydraulic properties of fractured media can dramatically change with scale.
\end{abstract}


In fractured rocks of very low matrix permeability, such as crystalline rocks, fluid flow is often restricted to a few fractures, as shown by hydraulic field experiments [1]. The occurrence of flow and transport in such fractured media is thus conditioned by the fracturenetwork connectivity at the system scale. The connectivity of fracture networks, which may be defined through the probability of connection of a system, has been determined for a variety of two- and three-dimensional elements. The early models using sticks in two dimensions [2,3] were followed by plates and polygons in three dimensions $[4,5]$, in order to account for more complex shapes of elements. According to more recent observations of fractured rocks, fractures are not only characterized by a variety of shapes but also by a broad range of lengths. Their length distribution is currently modeled by a power-law such as:

$$
n(l)=\alpha . l^{-a}
$$

where $n(l) . \mathrm{d} l$ is the number of fractures having a length in the range $[l, l+\mathrm{d} l], \alpha$ is the coefficient of proportionality, and $a$ is an exponent varying generally between 1 and 3 [6,7]. The consequences of this wide and non-limited range of fracture lengths on connectivity have been studied in two dimensions on off-lattice stick networks [8] as well as in three dimensions on on-lattice stick networks and on off-lattice networks of orthogonal planes [9]. In this paper, we propose an enhanced analysis of the connectivity properties of multi-scale fracture networks. Fractures are modeled by more realistic elliptic shapes [1] and the connectivity is analyzed for off-lattice networks of ellipses with widely-scattered distributions of eccentricity and length (Fig. 1).

In the scope of percolation theory, there is a single control parameter - the percolation parameter - that statistically measures the state of connection [10]. In short-range percolation, i.e. when all elements are much smaller than the system size, the control parameter is the density of objects. When systems are made up of elements of different shapes, the total excluded volume gives a better percolation criterion than the density of object and can thus be used as a better parameter of percolation [11].

However, because the natural fracture length distribution appears to be a power-law (1), fractures of the size of the system appear with a non-negligible probability and introduce long-range correlations. As a consequence, some systems may be connected at all scales by a single crossing fracture. The probability of including in the system such a fracture increases with scale provided that the length distribution is broad enough, implying that the density of objects at threshold, averaged over a large number of simulations, decreases with the system 
size. The density is thus no longer suitable for defining alone the connection state of the system. In this study, we show that there still exists a scale-invariant parameter for such longrange percolation problems, which is no longer the average of the mutual excluded volumes but their geometrical union.

\section{CLASSICAL EXPRESSION OF THE EXCLUDED VOLUME FOR A WIDELY- SCATTERED LENGTH DISTRIBUTION OF OBJECTS}

Because of its key role in this study, we give the detailed definition and expression of the total excluded volume as it has been classically derived firstly in short-range percolation and secondly in long-range percolation. In short-range percolation when all elements are identical, Balberg [11] defines the excluded volume of an object $\left(V_{\mathrm{e}}\right)$ "as the volume around an object into which the center of another similar object is not allowed to enter if overlapping of the two objects is to be avoided". The total excluded volume is this volume multiplied by the number of elements at threshold $N_{\mathrm{c}}$. For systems made up of non-identical elements, the total excluded volume $\left\langle V_{e x}\right\rangle$ is modified by replacing the mutual excluded volume $V_{e}$ by its average over all possible pairs of elements $\left\langle V_{e}\right\rangle$. The variations of $\left\langle V_{e x}\right\rangle$ at the percolation threshold with respect to the element shape remains limited so that $\left\langle V_{e x}\right\rangle=0.7-2.8$ in three dimensions $[12,13]$. In long-range percolation, the total excluded volume has been classically derived according to the same two-stages method: (i) the calculation of the mutual excluded volume $V_{\mathrm{e}}$ for two ellipses of different size and (ii) the average over all possible pairs of ellipses to get the total excluded volume $\left\langle V_{e x}>\right.$.

(i) The mutual excluded volume $V_{e}$ for any two convex overlapping objects can be derived theoretically as it depends only on the mean radii of curvature $R_{1}$ and $R_{2}$, on the surface areas $A_{1}$ and $A_{2}$ and on the volumes of the objects $V_{1}$ and $V_{2}[14]$ :

$$
\mathrm{V}_{\mathrm{e}}=\mathrm{V}_{1}+\mathrm{V}_{2}+\frac{\mathrm{A}_{1} \cdot \mathrm{R}_{2}+\mathrm{A}_{2} \cdot \mathrm{R}_{1}}{4 \pi}
$$

The expression of the mutual excluded volume of two discs of radii $R$ has been analytically calculated and is: $V_{e}=\pi^{2} \cdot R^{3}$ [4]. In the more complex case of ellipses of eccentricities $e_{1}$ and $e_{2}$ and of major-axis length $l_{1}$ and $l_{2}$, we computed the excluded volume numerically following the procedure of [13] and found:

$$
\mathrm{V}_{\mathrm{e}}=\pi^{2} \frac{e_{1} l_{1}^{2} l_{2}+e_{2} l_{1} l_{2}^{2}}{2}
$$


This last expression (3) is of the form of (2) and generalizes the expression of the mutual excluded volume of two discs.

(ii) When averaged over all possible pair of ellipses having both a distribution of eccentricities $e$ and major axes $l$, expression (3) leads to $\left\langle V_{e}>=\pi^{2} .<e>\right.$. $\left\langle l^{2}>\right.$. $<l>$. For a network made up of $N$ elements, the total excluded volume is simply the mutual excluded volume multiplied by $N$ [11]:

$$
<V_{e x}>=\pi^{2} . N .<e>.<l^{2}>.<l>
$$

In order to test our algorithms, we have computed $\left\langle V_{\mathrm{ex}}>\right.$ for systems of volume $V$ made up of discs having all the same radius much smaller than the system size, i.e. when the exponent $a$ in equation (1) tends toward infinity. We find a normalized total excluded volume $<V_{e x}>/ V$ of 2.2 which is in close agreement with [5] but different from $[13,15]$. The discrepancy of $20 \%$ with Charlaix's result is likely to result from the different way of generating the Poisson distribution of the disc centers.

When applied to systems having widely-scattered distributions of element size such that their power-law length exponent $a$ in expression (1) is lower than 4, Equation (4) normalized by the system volume was found to vary over several orders of magnitude at the percolation threshold (Fig. 2). This conclusion confirms the results previously obtained for perpendicular plans networks [9]. Because of its scale-dependence, the normalized total excluded volume cannot be used as a single order parameter for defining the state of connection of the system as it is in short-range percolation theory. However it is still exactly proportional to the density of intersections $I$ (Fig. 3). The number of intersections per ellipse is given by the probability of intersection between two ellipses $\left\langle V_{e}\right\rangle / V$ times the number of ellipses $N$, which leads to $I=<V_{\mathrm{ex}}>/ V$. As a consequence, at threshold, the number of intersections per object is no longer scale invariant as in short-range percolation [16]; more precisely it decreases by orders of magnitude. It especially implies that the density of interconnected objects at percolation threshold decreases with scale.

\section{EXPRESSION OF THE PARAMETER OF PERCOLATION FOR A WIDELY- SCATTERED LENGTH DISTRIBUTION OF OBJECTS}

Previous numerical results obtained on orthogonal plans seem to indicate that a scaleinvariant expression is given by the third moment of the length distribution $\left\langle l^{3}>\right.$ rather than by the multiplication of the first moment by the second moment $\left\langle l>.<l^{2}\right\rangle[4,9]$. We denote this new expression by $\left\langle V^{*}>\right.$ and derive it by replacing $\left\langle l>\right.$. $<l^{2}>$ by $\left\langle l^{3}\right\rangle$ in (4): 


$$
<V^{*}>=\pi^{2} . N .<e>.<l^{3}>
$$

We have checked that, whatever the length distribution, $\left\langle V^{*}\right\rangle / V$ remain scale-invariant for systems made up of discs (Fig. 4a) or of ellipses having either constant eccentricity or a uniform distribution of eccentricity (Fig. 4b). Because of its scale-invariance, $V^{*} / V$ can be used as the parameter of percolation $p$, proving meanwhile that the state of connection of the system can still be characterized by a single order parameter. We thus propose the following expression for the parameter of percolation $p$ :

$$
p=\pi^{2} \cdot N .<e>\cdot \frac{<l^{3}>}{L^{3}}
$$

where $L$ is the system size.

We note that the two expressions (4) and (5) normalized by the system volume $V$, i.e. $<V_{\mathrm{ex}}>/ V$ and $\left\langle V^{*}>/ V\right.$, lead approximately to the same estimate, as long as the length distribution remains bounded and narrow (i.e. for power-law length distributions such that $a>4$ ). On the other hand, when the radius distribution is widely-scattered -i.e. when $a<4$ in the case of the power-law distribution-, the two formulae lead to very different estimates. It especially means that the right theoretical expression can only be found by studying systems with widely-scattered length distribution.

\section{RELATION BETWEEN THE CONCEPT OF EXCLUDED VOLUME AND THE EXPRESSION OF THE PARAMETER OF PERCOLATION}

We have seen in the previous section that the classical total excluded volume is no longer connected to the parameter of percolation. In this section, we show that the parameter of percolation $p$ as given by (6) has still a sense in terms of mutual excluded volume. We argue that the volume $\left\langle V^{*}\right\rangle$ used in the derivation of the parameter of percolation for a set of $N$ ellipses is the union of the mutual excluded volume of the $N$ ellipses, whereas the total excluded volume $\left\langle V_{\mathrm{ex}}>\right.$ is the average of the mutual excluded volume over all possible pairs taken in the set formed by the $N$ ellipses.

We calculate the contribution of the $N$ ellipses to the excluded-volume union by ordering them in decreasing size. The contribution of the largest ellipse to the excludedvolume union, along with all other discs, is given precisely by the excluded volume of the two largest elements of sizes $l$ and $l$-d $l$, because all other mutual excluded volumes are imbedded in the excluded volume of these two largest elements. From the expression of the mutual excluded volume (3) and when $\mathrm{d} l<<$, the contribution of the two largest elements is given by 
$\pi^{2} .<e>. l^{3}$. We then remove the largest element from the system in order to avoid redundancy and calculate the contribution of the remaining ellipses to the excluded-volume union. This process iterated over all elements of decreasing size corresponds to the integration over the length distribution of ellipses, so that the excluded-volume union is equal to $\pi^{2} \int l^{3} . n(l) \cdot \mathrm{d} l$, which is precisely $\left\langle V^{*}\right\rangle$. We thus demonstrate that the parameter of percolation is the geometrical union of the mutual excluded volume.

The geometrical derivation of the excluded-volume union is closely related to the theoretical concept of percolation according to which the percolation threshold is reached when the available volume in terms of excluded volume vanishes. Moreover, the excludedvolume union $\left\langle V^{*}\right\rangle$ can be dominated by the largest elements, reflecting the fact that the network can be connected by the largest elements. By averaging the mutual excluded volume over all possible pairs of elements, the total excluded volume $\left\langle V_{\mathrm{ex}}\right\rangle$ remove this possibility and thus underestimates the connection probability of the system.

\section{ESTIMATES OF THE PERCOLATION THRESHOLD $P_{\mathrm{c}}$ AND GEOLOGICAL IMPLICATIONS}

In this section, we use the expression of the percolation parameter $p$ (6) to determine the percolation threshold $p_{c}$ as a function of the eccentricity $e$ and of the power-law length exponent $a$ (Figs. 5a and 5b). In the case of short-range percolation-i.e. for power-law length exponents $a$ larger than 4-, the values of $p_{\mathrm{c}}$ remain within the range 0.7-2.8 found by Balberg [12] (Fig. 5a). More generally, whatever the length and eccentricity distributions, the values of $p_{\mathrm{c}}$ remain restricted to a range of width smaller than one order of magnitude, giving in turn a first-order criterion of percolation for systems made up of widely-scattered length and aspect-ratio distributions.

The variations of $p_{\mathrm{c}}$ according to $e$ and $a$ display two common characteristics: (i) an increase when decreasing $a$ at fixed eccentricity $e$ (Fig. 5b) and (ii) a maximum when varying the eccentricity $e$ at fixed power-law length exponent $a$ (Fig. 5a). As this topic is still under investigations, we report here the most likely origins of these variations. (i) The increase of the percolation threshold $p_{\mathrm{c}}$ when the power-law length exponent $a$ decreases (Fig. 5b) may be due to truncation effects. Indeed ellipses truncated by the sides of the system (see Fig. 1) have an internal characteristic length smaller than the original one, and an aspect ratio larger than the generic eccentricity $e$. Since the probability of occurrence of large truncated fractures increases when $a$ decreases, we expect deviation due to truncation effect to increase when $a$ decreases. (ii) For networks made up of elements having all the same length much smaller 
than the system size $(a=\infty)$, the presence of the maximum may be an effect of the varying aspect ratio. The value of $p_{c}(e, a=\infty)$ is thought to increase when the local anisotropy decreases from that of sticks to that of spheres [11]. Applying this reasoning to ellipses in $3 \mathrm{D}$, the maximum value of the percolation parameter at threshold is attained when the local anisotropy is at a minimum. The local anisotropy is a measure of the dispersion of the typical lengths of the ellipse in the three dimensions:1,e,0. Since the minimum variance of this triplet is reached when $e=0.5$, the total excluded volume is expected to reach a maximum value for ellipses of eccentricity around 0.5 .

The parameter of percolation $p$ as defined by expression (6) can be derived analytically in the case of a power-law length distribution such as (1). As long as $a<4$, the parameter of percolation depends on the system size as $L^{4-a}$ for a given network (i.e. for fixed values of $a$ and fracture density). This case is relevant to natural fractures whose power-length exponents $a$ are in the range 2.5-4 [17]. The percolation parameter is thus expected to increase with system size, implying that systems are on average unconnected at small scales, and connected at large scales. The crossover scale above which fracture networks are always well connected is defined by $p(L)=p_{\mathrm{c}}$. This has a strong impact on the hydraulic properties of geological media. If, at low scales, the crystalline rocks permeability is very low because it is controlled by the matrix (non fractured rock), at scales larger than the previously identified crossover scale, there is an interconnected network of fractures, whose permeability can be larger by several orders of magnitude as observed by Clauser [18]. Similar conclusions have been drawn in clayey media [19]. The widely-scattered fracture-length distribution entails an increase of connectivity that in turn may change completely the hydraulic properties of the fractured media.

In conclusion, we have shown that the state of connection of a system made up of elements having a widely-scattered and non-limited length distribution is still determined by a single scale-invariant control parameter that we call, like in short-range percolation, the parameter of percolation. This parameter of percolation has a meaning in terms of excluded volume as it is precisely the geometrical union of the mutual excluded volumes. On the other hand, the total excluded volume -average of the mutual excluded volume over all pair of elements-, previously proposed as a parameter of percolation, decreases by orders of magnitude at threshold but still relates to the number of intersections. Fluctuations of the percolation threshold with the eccentricity and length distributions remain limited to a range smaller than one order of magnitude, giving thus a first rough percolation criterion. Finally, 
the new expression of the parameter of percolation underlines an increase of the connectivity of natural fractured media, which is consistent with the observed increase of permeability in fractured media. 


\section{Figure captions}

FIG. 1: Infinite clusters at threshold for $a=3.25$ and (a) $e=1$ (563 discs) for the network on the left and (b) $e=0.1$ (4900 ellipses) for the network on the right. The grey scale shading is proportional to the size of the element.

FIG. 2: $\left\langle V_{\mathrm{ex}}>/ V\right.$ at threshold normalized by the excluded volume at the minimal size $L_{0}$ for $a=\infty$ (squares), $a=4$ (circles), $a=3$ (upward triangles) and $a=2.5$ (downward triangles).

FIG. 3: Relation between the number of intersections and the total excluded volume $I /\left[<V_{\mathrm{ex}}>/ V\right]$ at threshold normalized by its value at the minimal length $L_{0}$ in the case of discs for $a=\infty$ (upward triangles), $a=3.5$ (circles) and $a=2.5$ (squares).

FIG.4: Scale invariance of $V^{*} / V$ for different length distributions (a) in the case of discs and (b) in the case of ellipses having fixed eccentricities (solid symbols) or a uniform distribution of eccentricities (open symbols).

FIG. 5: (a) Percolation threshold $p_{\mathrm{c}}$ against the eccentricity $e$ for $a=2.5$ (open discs), $a=3$ (upward open triangles), $a=3.5$ (downward open triangles), $a=5$ (open squares) and $a=\infty$ (solid squares). (b) Percolation threshold $p_{\mathrm{c}}$ against the power-law length exponent $a$ for different values of the eccentricity $e$. Dashed lines indicate the limits found by Balberg [12] for three-dimensional networks.

Acknowledgements- This work was supported by the European Community through the project SCALFRAC (Scale Dependence of Groundwater Flow and Contaminant Transport in Fractured Rock, Contract ENV4-CT97-0456) and the GdR ForPro. 


\section{References}

[1] National Research Council, Rock Fractures and Fluid Flow (National Academy Press, Washington, D.C., 1996).

[2] P. C. Robinson, J. Phys. A 16, 605 (1983).

[3] P. C. Robinson, J. Phys. A 17, 2823 (1984).

[4] E. Charlaix, E. Guyon, and N. Rivier, Solid State Commun. 50, 999 (1984).

[5] O. Huseby, J.-F. Thovert, and P. M. Adler, J. Phys. A 30, 1415 (1997).

[6] P. Davy, J. Geophys. Res. 98, 12 (1993).

[7] N. E. Odling, J. Struct. Geol. 19, 1257 (1997).

[8] O. Bour and P. Davy, Water Resour. Res. 33, 1567 (1997).

[9] O. Bour and P. Davy, Water Resour. Res. 34, 2611 (1998).

[10] D. Stauffer and A. Aharony, Introduction to percolation theory, second edition (Taylor and Francis, Bristol, 1992).

[11] I. Balberg, C. H. Anderson, S. Alexander, and N. Wagner, Phys. Rev. B 30, 3933 (1984).

[12] I. Balberg, Phys. Rev. B 31, 4053 (1985).

[13] E. J. Garboczi, K. A. Snyder, J. F. Douglas, and M. F. Thorpe, Phys. Rev. E 52, 819 (1995).

[14] A. Isihara, J. Chem. Phys. 18, 1446 (1950).

[15] E. Charlaix, J. Phys. A 19, L533 (1986).

[16] C. Baudet, E. Charlaix, E. Clément, E. Gyron, J. Hulin, and C. Leroy, in NATO Conference on "Scaling in Disordered Systems", edited by R. Pynn (Plenum, New-York, Geilo, Norway, 1985), p. 399.

[17] A. Nicol, J. J. Walsh, J. Watterson, and P. A. Gillepsie, J. Struct. Geol. 18, 191 (1996).

[18] C. Clauser, Eos Trans. AGU 73, 237 (1992).

[19] J. S. Hanor, Water Resour. Res. 29, 3691 (1993). 
(a)

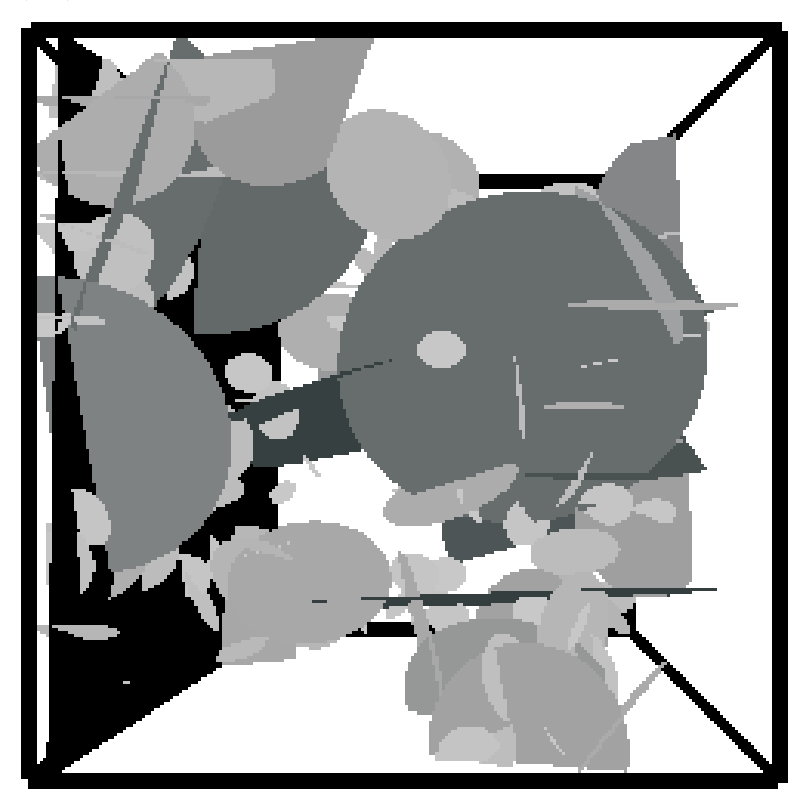

(b)

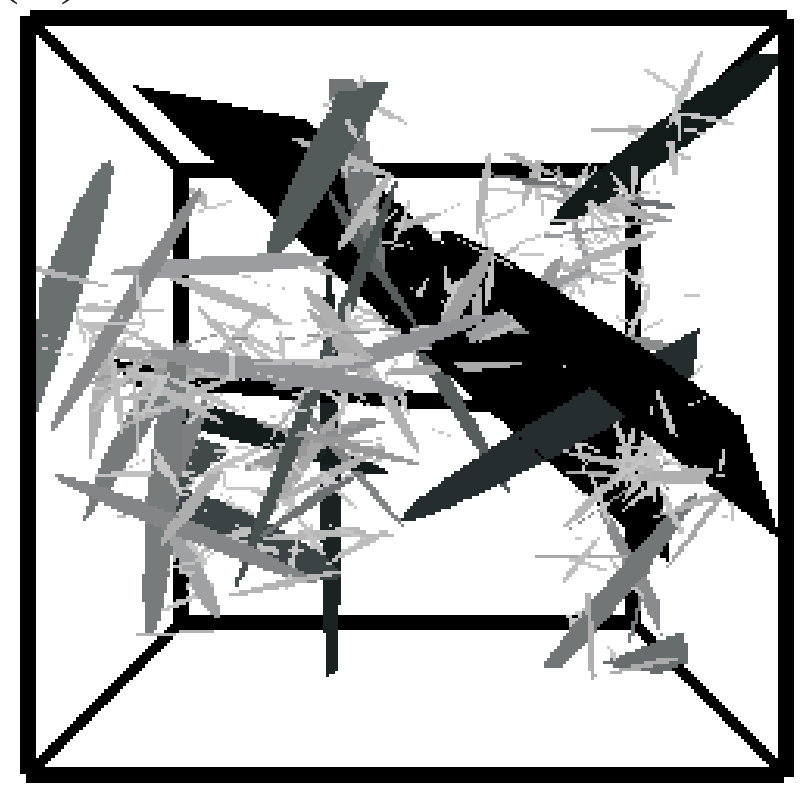




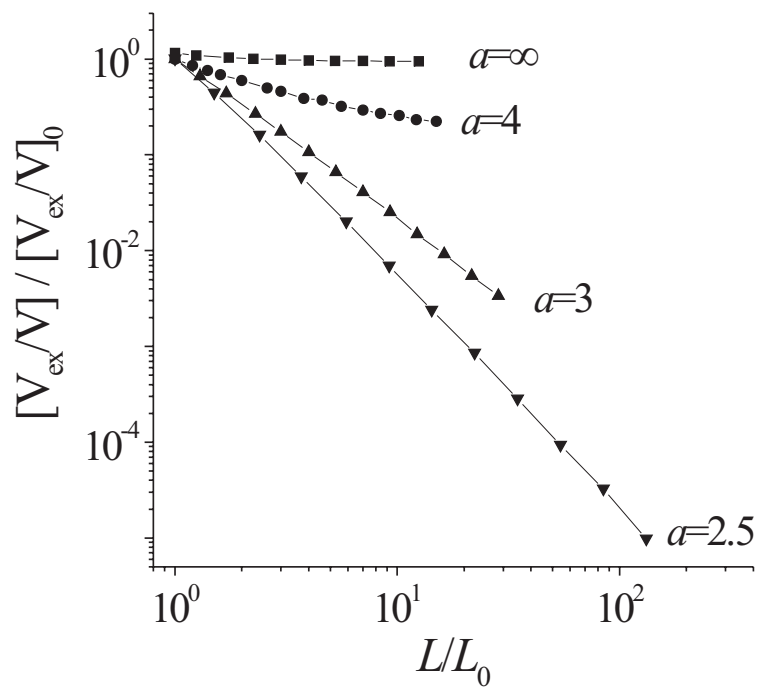




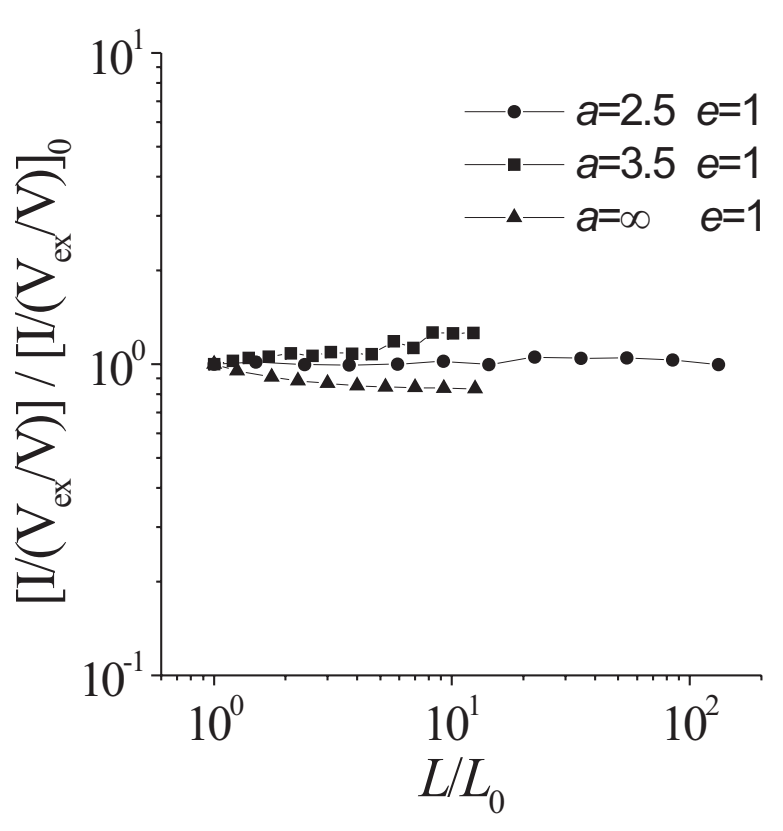


(a)

(b)
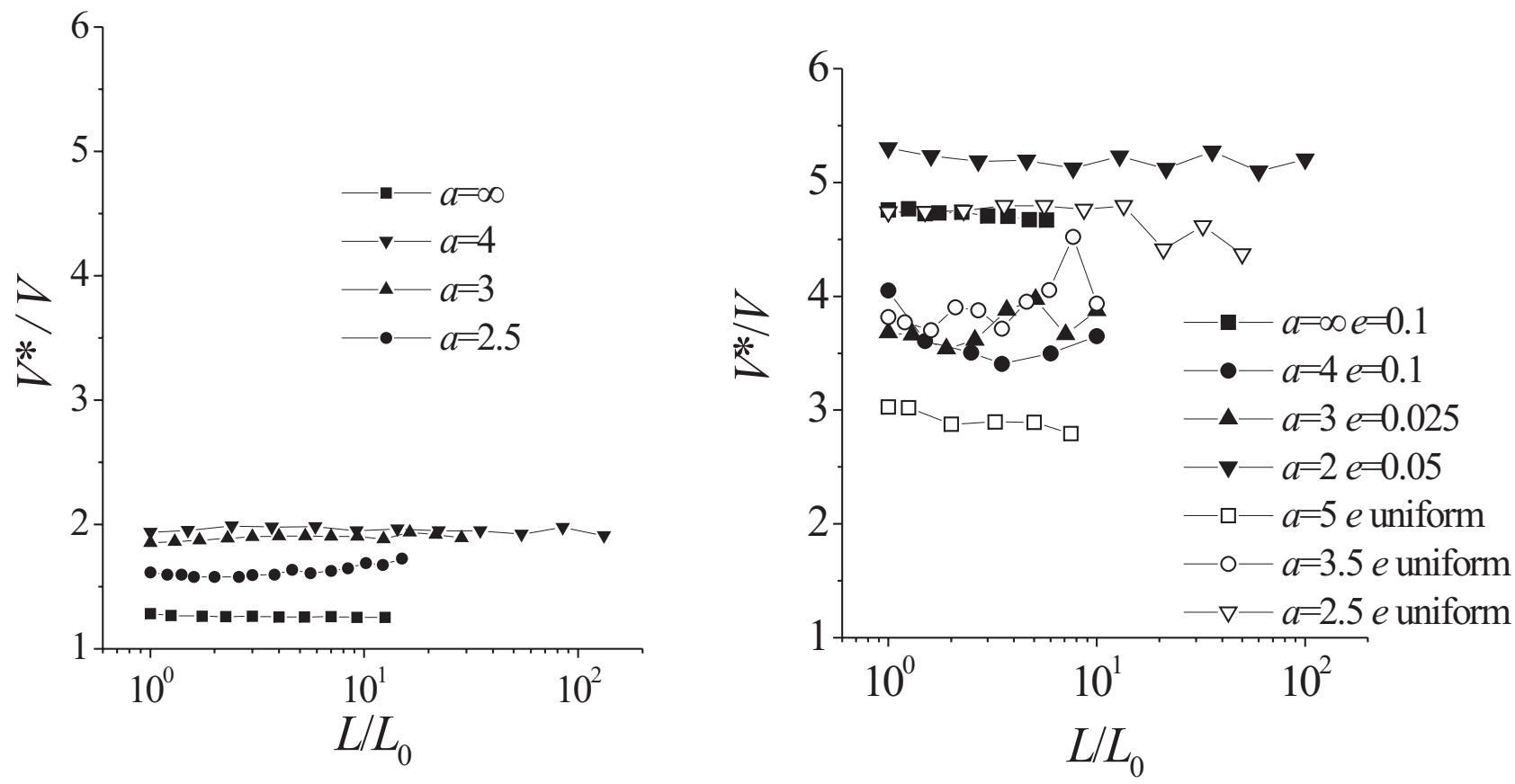
(a)

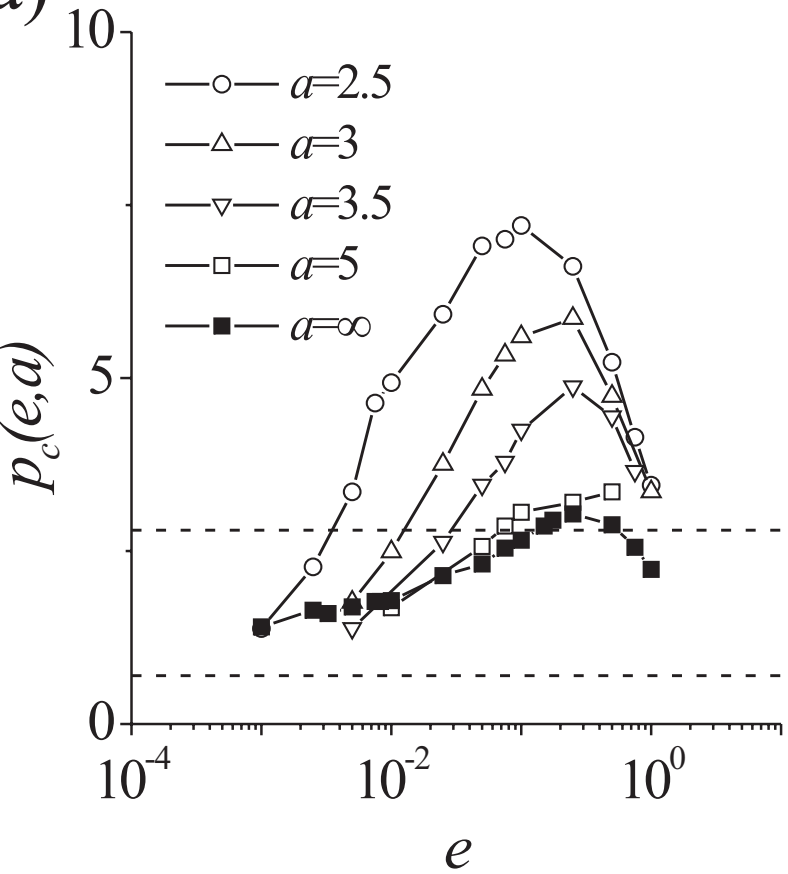

(b)

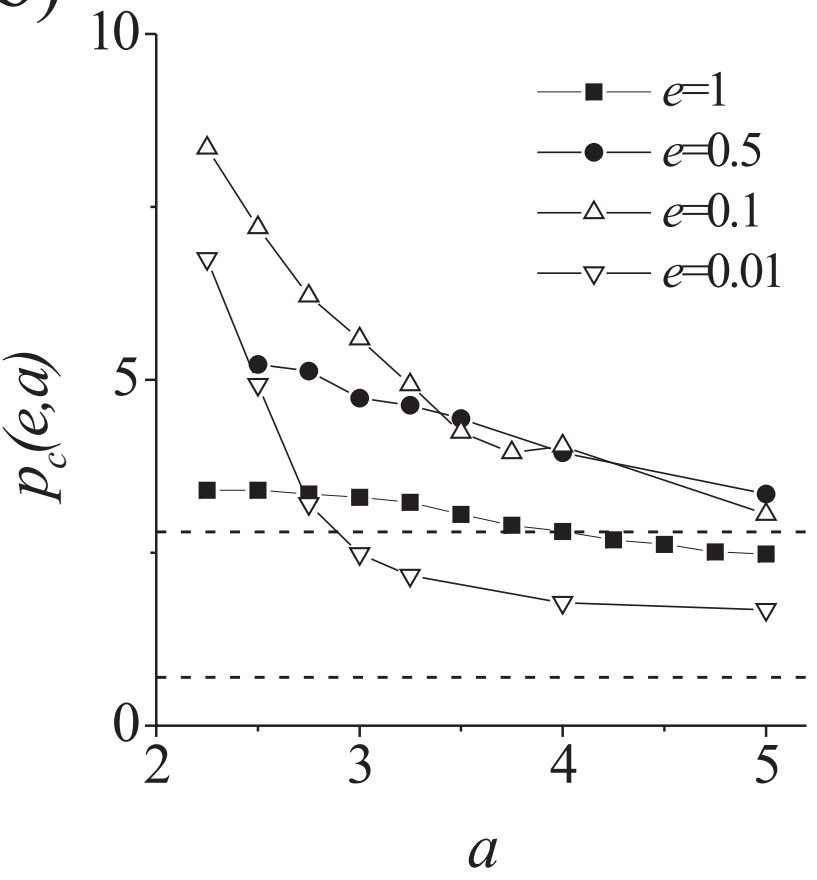

